\title{
Satisfaction with Subcutaneous Golimumab and its Auto-Injector among Rheumatoid Arthritis Patients with Inadequate Response to Adalimumab or Etanercept
}

\author{
Raphael J. Dehoratius ${ }^{1,2} \cdot$ Lawrence H. Brent $^{3} \cdot$ Jeffrey R. Curtis $^{4,6}$ • \\ Lorie A Ellis ${ }^{1} \cdot$ Kezhen L. Tang ${ }^{5}$
}

Published online: 9 February 2018

(C) The Author(s) 2018. This article is an open access publication

\begin{abstract}
Background Patient perceptions of treatment success, including satisfaction/preference, may complement clinical efficacy assessments.

Objective Our objective was to evaluate satisfaction with subcutaneous golimumab and its auto-injector in patients with rheumatoid arthritis (RA) and an inadequate adalimumab/etanercept response.

Methods In the multicenter, assessor-blinded GO-SAVE study, 433 patients with active RA (28-joint Disease Activity Score incorporating erythrocyte sedimentation rate $[\mathrm{DAS} 28$-ESR] $\geq 3.6$ and six or more swollen and six or more tender joints) despite methotrexate and past
\end{abstract}

Electronic supplementary material The online version of this article (https://doi.org/10.1007/s40271-018-0297-5) contains supplementary material, which is available to authorized users.

Jeffrey R. Curtis

jcurtis@uab.edu

1 Janssen Scientific Affairs, Horsham, PA, USA

2 Sidney Kimmel Medical College at Thomas Jefferson University, Philadelphia, PA, USA

3 Lewis Katz School of Medicine, Temple University, Philadelphia, PA, USA

4 University of Alabama at Birmingham, Birmingham, AL, USA

5 Janssen Research and Development, Spring House, PA, USA

6 University of Alabama at Birmingham, Faculty Offices Tower, Room 802, 510 20th Street South, Birmingham, AL 35294, USA adalimumab/etanercept treatment received open-label subcutaneous golimumab $50 \mathrm{mg}$ every 4 weeks ( $44 \mathrm{w}$ ) through week 12. Week 16 responders (DAS28-ESR improvement from baseline $>1.2$ and score $\leq 3.2$ ) continued therapy through week 52 ; nonresponders were randomized (1:2) to double-blind subcutaneous golimumab $50 \mathrm{mg} \mathrm{q} 4 \mathrm{w}$ or intravenous golimumab $2 \mathrm{mg} / \mathrm{kg}$ [weeks 16 , 20 , every 8 weeks $(q 8 w)]$. Patients rated satisfaction with their injection experience on a 5 -point scale $(1=$ very dissatisfied; $5=$ very satisfied) at screening, week 8 (all enrolled patients), and week 44 (for patients continuing open-label subcutaneous golimumab $50 \mathrm{mg} \mathrm{q} 4 \mathrm{w}$ ). Discomfort, pain, stinging, burning, and redness related to injection were assessed (none, mild, moderate, severe).

Results Similar proportions of patients $(N=433)$ had most recently received adalimumab $(50.3 \%)$ or etanercept $(49.7 \%)$ prior to golimumab. Overall satisfaction (somewhat/very) with the golimumab injection experience was reported by $84.4 \%$ of patients at week 8 versus $63.4 \%$ of patients who were satisfied with prior adalimumab/etanercept. Patients receiving open-label subcutaneous golimumab through week $44(N=75)$ reported much less discomfort $(60.9 \%)$, redness $(60.9 \%)$, pain $(59.4 \%)$, stinging $(67.2 \%)$, and burning (65.6\%) with the golimumab injection than with their previous tumor necrosis factor antagonist medication injection.

Conclusion Most patients with RA receiving golimumab following adalimumab/etanercept inadequate response were satisfied with their overall golimumab experience, including its auto-injector versus their previous injection device.

Clinical trials.gov NCT01004432; EudraCT 2009-010582-23. 


\section{Key Points for Decision Makers}

Patient preferences can be indicators of treatment success and quality.

The golimumab GO-SAVE trial employed active surveillance to comparatively examine patient satisfaction among biologic products in rheumatoid arthritis (RA).

Most patients with RA who transitioned to golimumab from adalimumab or etanercept were satisfied with their overall golimumab experience.

\section{Introduction}

Patient satisfaction with medical treatments may complement more traditional, physician-based, clinical measures of effectiveness. Studies in a wide variety of therapeutic areas have demonstrated associations between greater patient satisfaction with their medical treatments and better physical health status, less disability, and greater quality of life [1]. Patients who are more satisfied with their treatment may be more engaged in their treatment and thus more adherent [2].

Specific to rheumatoid arthritis (RA), the availability of biologic agents has expanded the options for route and timing of administration as well as the range of possible toxicities and cost of therapy [3]. These factors can influence a patient's overall satisfaction with their treatment, as mode and frequency of treatment administration are important to patients with RA. In a conjoint analysis of approximately 900 respondents with a self-reported physician diagnosis of moderate-to-severe RA, patients were willing to accept treatments with lower efficacy and greater safety risks to attain shorter treatment duration and less frequent administration [4]. Specifically, in their systematic quantification of marginal utilities, Poulos et al. [4] found that preference for a reduction in the duration of a quarterly infusion from 2 to $1 \mathrm{~h}$ was about five times greater than preference for an increase in treatment response rate of 1 percentage point (from 60 to $61 \%$ ) and was also 12 and 2 times greater than the preference for changes of 1 percentage point in the chance of mild or serious treatment reactions, respectively. Thus, together with the fact that injection experience is an often-cited reason for discontinuation of anti-tumor necrosis factor (TNF) medication by patients with RA [5], one can conclude that patients' perspectives should factor prominently in the selection of a treatment regimen.

Golimumab is a biologic agent approved for the treatment of RA. The fully human anti-TNF monoclonal antibody is available as a subcutaneous injection as well as by intravenous infusion. Following the approval of subcutaneous golimumab for the treatment of RA in 2009, the golimumab GO-SAVE trial was undertaken. This was a phase IIIb multicenter study evaluating the efficacy of subcutaneous and intravenous golimumab in patients with RA with inadequate disease control despite treatment with etanercept or adalimumab. This study was unique because it provided data comparing prior and current treatments related to patient-reported satisfaction with treatment administration, which are important as part of a patientfocused approach now common in rheumatology. A prior report detailed the efficacy and safety findings from the GO-SAVE trial [6]; we now report patient satisfaction with subcutaneous golimumab and its mode of administration in the trial participants with RA who had an inadequate response to prior adalimumab or etanercept.

\section{Patients and Methods}

\subsection{Patients and Trial Design}

The GO-SAVE trial (NCT01004432, EudraCT 2009-010582-23) was conducted according to the principles of the Declaration of Helsinki and International Committee on Harmonisation good clinical practices. The protocol was approved by each study site's governing ethical body, and patients provided written informed consent prior to the conduct of any study-related procedures.

Details of the GO-SAVE patient eligibility criteria have been reported [6]. Briefly, adult patients with active RA following an inadequate response to $\geq 3$ months of treatment with an approved stable regimen of etanercept (50 mg once weekly or $25 \mathrm{mg}$ twice weekly) or adalimumab (40 mg every 2 weeks) in combination with stable oral methotrexate $(\geq 7.5$ to $\leq 25 \mathrm{mg} /$ week for $\geq 4$ weeks) were included in the study. Active disease/ inadequate response was defined as a 28 -joint Disease Activity Score incorporating erythrocyte sedimentation rate $(\mathrm{DAS} 28-\mathrm{ESR}) \geq 3.6$ accompanied by at least 6 of 66 swollen and at least 6 of 68 tender joints. Patients entered the screening period 6 weeks before receiving golimumab (week -6) and continued their original anti-TNF therapy; their conformance with study eligibility criteria was reconfirmed at week 0 . All eligible patients were actively transitioned to receive open-label subcutaneous injections of golimumab $50 \mathrm{mg}$ at week 0 in combination with their stable oral methotrexate regimen and received injections 
every 4 weeks [Fig. 1a in the Electronic Supplementary Material (ESM).]

At week 16 after starting golimumab, response was assessed using the DAS28-ESR European League Against Rheumatism (EULAR) criteria, and patients with a good response (improvement from baseline $>1.2$ and a score $\leq 3.2$ ) [7] continued open-label subcutaneous golimumab $50 \mathrm{mg}$ plus methotrexate every 4 weeks through week 48 (group 1). All other patients, who were deemed to have an inadequate response (i.e., EULAR moderate or no response), were randomized $1: 2$ to receive double-blind subcutaneous golimumab every 4 weeks (group 2a) plus methotrexate or intravenous golimumab $2 \mathrm{mg} / \mathrm{kg}$ at weeks 16 and 20 and then every 8 weeks (group 2b) plus methotrexate through week 48 (Fig. 1a in the ESM). Further details of study blinding and conduct have been reported previously [6].

Patient satisfaction questionnaires were developed by study investigators to understand the different injection experience attributes reported by patients when treated with golimumab versus when treated with adalimumab or etanercept. Patient injection experience with adalimumab and etanercept was assessed at baseline (week -2 for patients receiving adalimumab, and week -1 for patients receiving etanercept, to align with the frequency of the dosing regimen). Patients were questioned on the device's ease of use (7-point scale ranging from "extremely difficult" to "extremely easy") and the level of discomfort, pain, stinging, burning, and redness associated with their most recent injection (none, mild, moderate, severe). Patients also rated their satisfaction (5-point scale ranging from "very dissatisfied" to "very satisfied") with the frequency of injection, injection device, and injection experience for either adalimumab or etanercept.

Patient injection experience with golimumab was assessed at week 8 for all patients and again at week 44 for patients continuing subcutaneous golimumab, as was medication and injection device preference for golimumab versus adalimumab or etanercept. Patients were asked to rate the level of discomfort, pain, stinging, burning, and redness associated with golimumab injection compared with that for their previous medication (5-point scale ranging from "much less" to "much more"). Patients also rated their satisfaction (5-point scale ranging from "very dissatisfied" to "very satisfied") with the frequency of injection, injection device, and injection experience for golimumab and indicated their preference for injection frequency, device, and medication. The golimumab device's ease of use (7-point scale ranging from "extremely difficult" to "extremely easy") was also assessed at week 44. Please see the ESM for further details of the patient satisfaction and preference questionnaires.

\subsection{Data Analyses}

Treatment satisfaction with golimumab versus prior antiTNF therapy was assessed overall and by prior anti-TNF drug (etanercept or adalimumab) or prior anti-TNF device type (pen or syringe) via Chi-square testing. Allowable syringe devices for etanercept included both the prefilled syringe and the lyophilized vial/syringe. The degree of agreement between week- 8 and week- 44 patient satisfaction and preference findings were evaluated using Kappa statistics.

All patient-reported satisfaction data were analyzed using a modified intent-to-treat (mITT) approach. During the 16-week open-label treatment period, the mITT population included the 433 enrolled patients who had baseline measurements and received at least one dose of open-label subcutaneous golimumab. During the continued open-label/double-blind treatment period (weeks 16-52), the treatment satisfaction and preference questionnaire was administered to patients who continued to receive openlabel subcutaneous golimumab. Thus, the mITT population during this timeframe included the 75 patients who received at least one dose of open-label subcutaneous golimumab. All analyses were performed using SAS version 9.2 .

\section{Results}

\subsection{Patient Disposition and Baseline Characteristics}

Patient disposition through week 52 of the GO-SAVE trial has been described previously [6]. Patients were enrolled by 131 international study sites, and the study was conducted from October 2009 through May 2013. From weeks $0-16,433$ patients received open-label subcutaneous golimumab 50 mg every 4 weeks, including 186 (43.0\%), 174 $(40.2 \%)$, and $73(16.9 \%)$ patients who had previously received adalimumab, etanercept, or both, respectively (Table 1).

In total, $83(19.2 \%)$ patients discontinued the study agent through week 16. At week 16, $75(17.3 \%)$ patients with a EULAR DAS28-ESR good response continued to receive open-label subcutaneous golimumab $50 \mathrm{mg}$ every 4 weeks (group 1), and $275(63.5 \%)$ patients were randomized to receive double-blind subcutaneous golimumab injections (group 2a; $n=91$ ) or intravenous golimumab infusions at weeks 16 and 20, and then every 8 weeks (group 2b; $n=184$ ), all through week 48 (Fig. $1 \mathrm{~b}$ in the ESM).

Among enrolled patients, the mean age was 55.7 years, mean DAS28-ESR was 6.24, and approximately $50 \%$ of the patients had been diagnosed with RA $>7$ years prior to study entry. Baseline patient and disease characteristics 
Table 1 Summary of baseline patient and characteristics; modified intent-to-treat population

\begin{tabular}{|c|c|c|c|}
\hline \multirow[t]{3}{*}{ Characteristics } & \multicolumn{3}{|c|}{ Golimumab $50 \mathrm{mg} \mathrm{SC}$} \\
\hline & \multirow[t]{2}{*}{ All patients } & \multicolumn{2}{|c|}{ Prior anti-TNF device } \\
\hline & & Pen & Syringe \\
\hline Patients $(N)$ & 433 & 239 & 194 \\
\hline Age (years) & $55.7 \pm 11.52$ & $54.3 \pm 11.21$ & $57.5 \pm 11.67$ \\
\hline \multicolumn{4}{|l|}{ Sex } \\
\hline Male & $75(17.3)$ & $39(16.3)$ & $36(18.6)$ \\
\hline Female & $358(82.7)$ & $200(83.7)$ & $158(81.4)$ \\
\hline Duration of rheumatoid arthritis (years) & $10.68 \pm 9.798$ & $9.23 \pm 9.324$ & $12.47 \pm 10.092$ \\
\hline$<2$ & $66(15.2)$ & $49(20.5)$ & $17(8.8)$ \\
\hline $2-7$ & $150(34.6)$ & $90(37.7)$ & $60(30.9)$ \\
\hline$>7$ & $217(50.1)$ & $100(41.8)$ & $117(60.3)$ \\
\hline DAS28-ESR & $6.24 \pm 0.937$ & $6.29 \pm 0.915$ & $6.17 \pm 0.961$ \\
\hline$\leq 5.1$ & $47(10.9)$ & $20(8.4)$ & $27(13.9)$ \\
\hline$>5.1$ & $386(89.1)$ & 219 (91.6) & $167(86.1)$ \\
\hline Number of tender joints (0-68) & $31.3 \pm 16.97$ & $32.0 \pm 17.15$ & $30.5 \pm 16.76$ \\
\hline Number of swollen joints (0-66) & $18.4 \pm 11.84$ & $19.2 \pm 12.36$ & $17.4 \pm 11.11$ \\
\hline HAQ-DI (0-3) & $1.36 \pm 0.657$ & $1.35 \pm 0.635$ & $1.38 \pm 0.685$ \\
\hline Erythrocyte sedimentation rate $(\mathrm{mm} / \mathrm{h})$ & $32.08 \pm 20.479$ & $33.05 \pm 21.055$ & $30.88 \pm 19.735$ \\
\hline C-reactive protein $(\mathrm{mg} / \mathrm{l})$ & $7.87 \pm 12.217$ & $7.46 \pm 10.933$ & $8.37 \pm 13.646$ \\
\hline \multicolumn{4}{|l|}{ Previous anti-TNF treatment } \\
\hline Etanercept only & $174(40.2)$ & $92(38.5)$ & $82(42.3)$ \\
\hline Adalimumab only & $186(43.0)$ & $110(46.0)$ & $76(39.2)$ \\
\hline Etanercept and adalimumab & $73(16.9)$ & $37(15.5)$ & $36(18.6)$ \\
\hline \multirow[t]{2}{*}{ Baseline adalimumab dose (mg SC EOW) } & 217 & 124 & 93 \\
\hline & $(40.0 \pm 0.00)$ & $(40.0 \pm 0.00)$ & $(40.0 \pm 0.00)$ \\
\hline \multirow[t]{2}{*}{ Baseline etanercept dose (mg/week) } & 215 & 114 & 101 \\
\hline & $(48.8 \pm 5.28)$ & $(49.8 \pm 2.34)$ & $(47.8 \pm 7.16)$ \\
\hline Baseline methotrexate dose (mg/week) & $16.4 \pm 5.23$ & $16.7 \pm 5.07$ & $16.0 \pm 5.41$ \\
\hline \multirow[t]{2}{*}{ Baseline corticosteroid dose (mg/day) } & 131 & 77 & 54 \\
\hline & $(5.6 \pm 2.46)$ & $(6.0 \pm 2.39)$ & $(5.1 \pm 2.49)$ \\
\hline
\end{tabular}

Data presented are mean \pm standard deviation, $N$ (mean \pm standard deviation) or $N(\%)$ unless noted otherwise

DAS28-ESR 28-joint disease activity score incorporating erythrocyte sedimentation rate, EOW every other week, $H A Q-D I$ health assessment questionnaire-disability index, $S C$ subcutaneous, TNF tumor necrosis factor

were generally consistent between patients whose prior anti-TNF agent was administered via pen versus syringe, although patients with prior syringe use appeared to have more longstanding disease (Table 1).

\subsection{Clinical Efficacy}

As reported previously [6], 151 of 433 patients [34.9\%; 95\% confidence interval (CI) 30.4-39.4] who transitioned from adalimumab or etanercept to subcutaneous golimumab achieved the study's primary endpoint at week 14, i.e., at least $20 \%$ improvement in the American College of Rheumatology criteria (ACR20 response) from baseline ( $p<0.001$ for rejecting the main trials' null hypothesis that the proportion of patients with an ACR20 response would be $\leq 0.20$ ). Additionally, among patients who achieved a DAS28-ESR good response at week 16 and continued open-label subcutaneous golimumab, 22.7\% (17/75) maintained their response through week 52 [6].

\subsection{Prior Anti-Tumor Necrosis Factor Medication and Injection Experiences}

Based on the most recent biologic received, similar proportions of patients had received adalimumab $(50.3 \%$, $218 / 433)$ or etanercept $(49.7 \%, 215 / 433)$. Prior injection device experience also was generally evenly distributed among the 433 patients, with 125 (28.9\%) having 
experience with the adalimumab pen, $93(21.5 \%)$ with the adalimumab prefilled syringe, $114(26.3 \%)$ with the etanercept SureClick pen, and 93 (21.5\%) with the etanercept prefilled syringe. Eight patients $(1.8 \%)$ had most recently received etanercept via vial and syringe.

Most patients $(78.3 \%, 339 / 433)$ self-injected their previous anti-TNF medication and reported mild or moderate (combined) stinging (73.9\%), discomfort and pain (each reported by $66.3 \%$ of patients), and burning $(59.8 \%)$. Mild or moderate redness was a less common side effect of the prior medication injection (38.3\%). Severe injection reactions associated with previous anti-TNF medication were reported by $<8 \%$ of patients for each of the five symptoms assessed by the satisfaction with injection experiencebaseline questionnaire (adalimumab week -2, etanercept week -1) (Fig. 1a). Patient experiences with prior anti-TNF agents are shown for patients who received the prior agent by syringe versus pen devices in Fig. 2a, b in the ESM.

\subsection{Auto-Injector Device: Satisfaction and Patient Preference}

At week 8, satisfaction ("somewhat" or "very") with the golimumab injection device was reported by $84.4 \%$ of patients (Fig. 2a) versus $63.4 \%$ (242/382) of patients who were satisfied with prior adalimumab/etanercept (Table 2). The golimumab auto-injector was preferred over the previous injectable device by $71.4 \%$ of patients (Fig. 3a in the ESM). The shift from baseline (previous anti-TNF medications) to week 8 (golimumab) in patient satisfaction with the injectable device is also shown in Table 2.

Preference for the golimumab auto-injector at week 8 was similar between patients who had used the adalimumab $(73.0 \%, 143 / 196)$ or etanercept $(69.7 \%, 131 / 188)$ injectable devices. However, the proportions of patients who were "very" satisfied with the golimumab injection device at week 8 were somewhat higher among those who had used the etanercept $(66.0 \%, 124 / 188)$ versus adalimumab $(60.2 \%, 118 / 196)$ device.

Overall, $68.9 \%$ of patients were "very" satisfied with the golimumab device (auto-injector) among the patients who used a pen versus $55.8 \%$ of patients who used a syringe for prior anti-TNF treatment $(p=0.0325)$ (Fig. 3b). Among patients who used an injection pen for prior antiTNF treatment, $75.0 \%$ preferred the golimumab device (auto-injector), $6.1 \%$ preferred the previous injection pen, and $18.9 \%$ had no preference (Fig. $4 \mathrm{~b}$ in the ESM). For patients who used a syringe for prior anti-TNF treatment, $66.9 \%$ preferred the golimumab device (auto-injector), $13.4 \%$ preferred the previous injection syringe, and $19.8 \%$ had no preference $(p=0.0444)$ (Fig. $4 \mathrm{a}$ in the ESM). Findings at week 44 were consistent with those at week 8 (Fig. 2b and Fig. 3b in the ESM). The consistency between
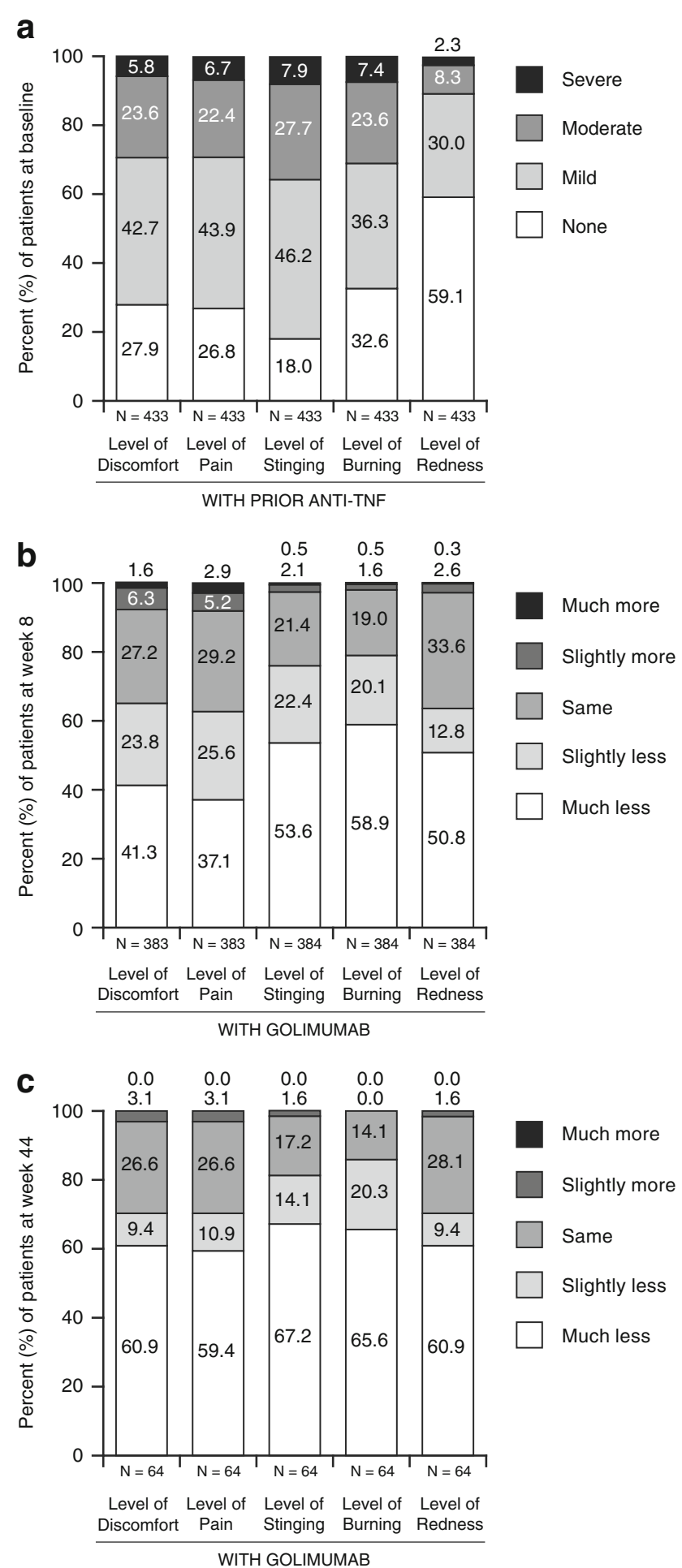

Fig. 1 Patient experience a with prior anti-TNF agent and with golimumab vs. prior anti-TNF agent (adalimumab at week -2/ etanercept at week -1) at b week 8 and $\mathbf{c}$ week 44. TNF Tumor necrosis factor

week 8 and week 44 golimumab auto-injector device satisfaction was further supported by a Kappa statistic of 0.42 (95\% CI $0.14-0.69 ; p=0.003)$. 
a

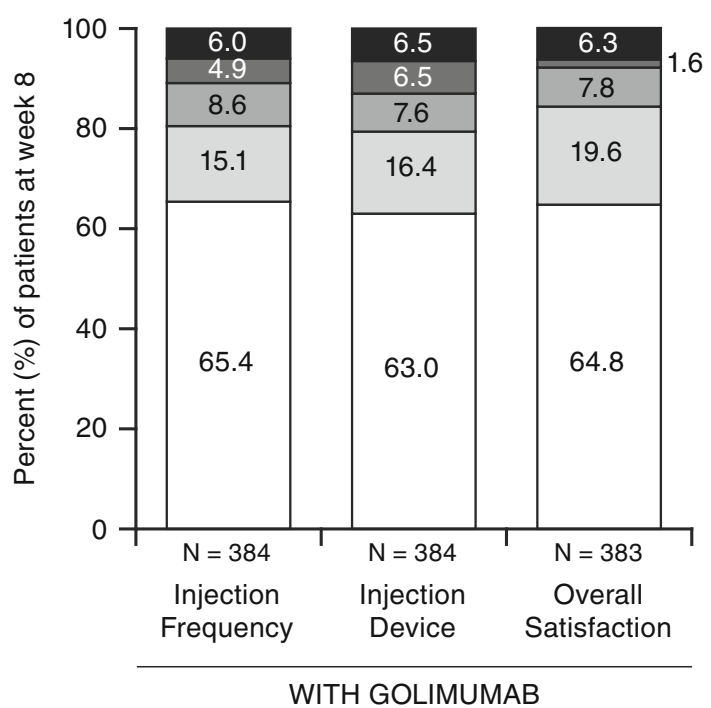

WITH GOLIMUMAB b

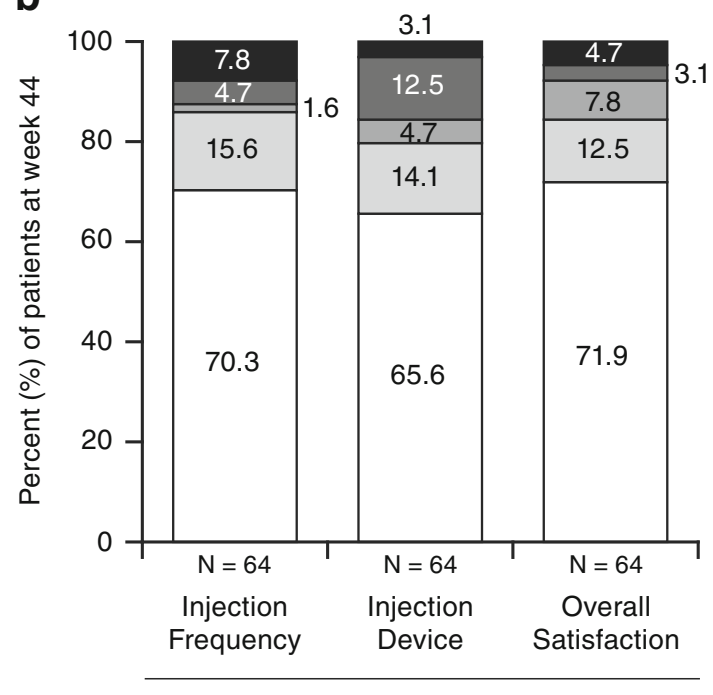

WITH GOLIMUMAB

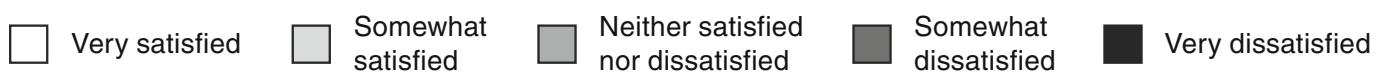

Fig. 2 Patient satisfaction with golimumab injection at a week 8 and b week 44

\subsection{Comparative Experience: Satisfaction and Patient Preference}

When comparing injection experiences between golimumab and their previous medicine (adalimumab or etanercept) at week 8 , a total of $41.3 \%$ of patients reported "much less" discomfort, $37.1 \%$ reported "much less" pain, $53.6 \%$ reported "much less" stinging, 58.9\% reported "much less" burning, and 50.8\% reported "much less" redness with the golimumab injection (Fig. 1b). Additionally, higher proportions of patients who previously received anti-TNF treatment via a pen device reported much or slightly less discomfort (66.3\%), pain (65.4\%), stinging (81.2\%), burning (83.5\%), and redness $(71.7 \%)$ (Fig. 2d in the ESM) than did patients whose prior antiTNF agent was given via a syringe $(63.4,59.3,69.8,73.2$, and $53.5 \%$, respectively) (Fig. 2c in the ESM).

At baseline, $51.0 \%$ of patients reported being "somewhat" or "very" satisfied with their previous injection frequency, whereas-at week 8-a total of $80.5 \%$ of patients were "somewhat" or "very" satisfied with the golimumab injection frequency (Fig. 2a). At week 8, overall satisfaction ("somewhat" or "very") with the golimumab injection experience was reported by $84.4 \%$ of patients (Fig. 2a), and $72.3 \%$ of patients preferred golimumab over their previous anti-TNF medication (Fig. 3b in the ESM). Patients ranked one injection per month at home as the most preferred dosing frequency at week 8 .
Baseline overall satisfaction ("somewhat" or "very") with injection experience was similar between patients who received etanercept $(65.1 \%, 140 / 215)$ or adalimumab $(61.9 \%, 135 / 218)$. However, the proportions of patients who reported "much less" discomfort (48.0 vs. 34.2\%), pain ( 44.9 vs. $28.9 \%)$, burning ( 62.2 vs. $55.3 \%)$, and redness (52.6 vs. $48.9 \%$ ) with the golimumab injection at week 8 appeared somewhat higher among patients who previously received adalimumab $(n=195$ or 196) versus etanercept ( $n=187$ or 188), respectively. Consistently, the proportions of patients who were "very" satisfied with the golimumab injection frequency $(70.2 \%$ etanercept vs. $60.7 \%$ adalimumab) and overall injection experience (71.1\% etanercept vs. $58.7 \%$ adalimumab) at week 8 appeared to be higher among those who most recently received etanercept versus adalimumab. However, overall, the proportions of patients who preferred golimumab over their prior anti-TNF medication were similar between patients who received adalimumab $(73.8 \%, 144 / 195)$ and those who received etanercept $(70.7 \%, 133 / 188)$.

A higher level of overall satisfaction with the golimumab injection experience at week 8 was also observed for patients who used the injection pen for prior anti-TNF treatment than those who used the syringe for prior antiTNF treatment, i.e., $72.0 \%$ of patients who used the injection pen versus $55.8 \%$ of patients who used the syringe for prior anti-TNF treatment were "very satisfied" with the golimumab injection at week $8(p=0.0076)$ 
Table 2 Treatment satisfaction: shift from baseline to week 8 in the modified intent-to-treat population $(n=382)$

\begin{tabular}{|c|c|c|c|c|c|}
\hline \multirow[t]{2}{*}{ Variable } & \multicolumn{5}{|c|}{ Baseline satisfaction with adalimumab (week -2)/etanercept (week -1) } \\
\hline & Very dissatisfied & Somewhat dissatisfied & $\begin{array}{l}\text { Neither satisfied } \\
\text { nor dissatisfied }\end{array}$ & Somewhat satisfied & Very satisfied \\
\hline \multicolumn{6}{|c|}{ Satisfaction with injection frequency at week 8} \\
\hline$N^{\mathrm{a}}$ & 22 & 58 & 109 & 94 & 100 \\
\hline Very dissatisfied & $1(4.5)$ & $5(8.6)$ & $5(4.6)$ & $4(4.3)$ & $8(8.0)$ \\
\hline Somewhat dissatisfied & 0 & $2(3.4)$ & $8(7.3)$ & $5(5.3)$ & $4(4.0)$ \\
\hline Neither satisfied nor dissatisfied & $2(9.1)$ & $5(8.6)$ & $11(10.1)$ & $10(10.6)$ & $4(4.0)$ \\
\hline Somewhat satisfied & $2(9.1)$ & $8(13.8)$ & $21(19.3)$ & $14(14.9)$ & $13(13.0)$ \\
\hline Very satisfied & $17(77.3)$ & $38(65.5)$ & $64(58.7)$ & $61(64.9)$ & $71(71.0)$ \\
\hline \multicolumn{6}{|c|}{ Satisfaction with the injectable device at week 8} \\
\hline$N^{\mathrm{a}}$ & 30 & 30 & 62 & 102 & 158 \\
\hline Very dissatisfied & $3(10.0)$ & $4(13.3)$ & $3(4.8)$ & $5(4.9)$ & $10(6.3)$ \\
\hline Somewhat dissatisfied & $3(10.0)$ & 0 & $4(6.5)$ & $8(7.8)$ & $10(6.3)$ \\
\hline Neither satisfied nor dissatisfied & $1(3.3)$ & $1(3.3)$ & $8(12.9)$ & $6(5.9)$ & $13(8.2)$ \\
\hline Somewhat satisfied & $6(20.0)$ & $4(13.3)$ & $14(22.6)$ & $21(20.6)$ & $17(10.8)$ \\
\hline Very satisfied & $17(56.7)$ & $21(70.0)$ & $33(53.2)$ & $62(60.8)$ & $108(68.4)$ \\
\hline \multicolumn{6}{|c|}{ Overall satisfaction with injection experience at week 8} \\
\hline$N^{\mathrm{a}}$ & 19 & 35 & 86 & 119 & 123 \\
\hline Very dissatisfied & 0 & $6(17.1)$ & $5(5.8)$ & $5(4.2)$ & $8(6.5)$ \\
\hline Somewhat dissatisfied & 0 & 0 & $1(1.2)$ & $3(2.5)$ & $2(1.6)$ \\
\hline Neither satisfied nor dissatisfied & $1(5.3)$ & $3(8.6)$ & $12(14.0)$ & $5(4.2)$ & $9(7.3)$ \\
\hline Somewhat satisfied & $3(15.8)$ & $5(14.3)$ & $22(25.6)$ & $25(21.0)$ & $20(16.3)$ \\
\hline Very satisfied & $15(78.9)$ & $21(60.0)$ & $46(53.5)$ & $81(68.1)$ & $84(68.3)$ \\
\hline
\end{tabular}

Data are presented as $N(\%)$ unless otherwise indicated

${ }^{a}$ Includes only patients who had provided the answer at both baseline and week 8 for a given question

(Fig. 3). When further evaluating the injection experience by injection frequency, no differences between prior pen and syringe use were observed $(p=0.1761)$ (Fig. 3). Compared with prior medication, $74.1 \%$ of patients who used the injection pen for prior anti-TNF treatment preferred golimumab, $3.3 \%$ preferred their previous medication, and $22.6 \%$ had no preference. Similar findings were derived from patients who used a syringe for prior antiTNF treatment ( $p=0.6360$; Fig. 4 in the ESM). Consistent findings were generally observed at week 44 (Figs. 1c and $2 b$, and Fig. $3 b$ in the ESM), although the Kappa statistics for agreement between week 8 and week 44 in terms of treatment frequency and overall satisfaction were $<0.2$ and not significant $(p>0.15)$, due to $11 \%(7 / 63)$ and $6.5 \%(4 / 62)$ of patients who shifted response from satisfied to dissatisfied for treatment frequency and overall satisfaction, respectively, and 9.5\% (6/63) and 6.5\% (4/62) of patients who shifted response from dissatisfied to satisfied between week 8 and week 44 for treatment frequency and overall satisfaction, respectively. Nonetheless, most patients were consistently satisfied with injection frequency and overall injection experience at weeks 8 and 44 .

\section{Discussion}

As early as 2001, the Institute of Medicine identified the concept that healthcare should be "patient-centered" in its recommendations to improve the quality of healthcare delivery systems. While it did not include patient satisfaction as part of such quality measures, patient satisfaction questionnaires are now commonly employed by payers and hospitals in the USA to measure value in healthcare [8]. More recently, the Patient Protection and Affordable Care Act of 2010 has required the development of a patient satisfaction survey system as part of its quality rating system $[9,10]$. Given that patient experience now serves as a measure of healthcare quality and value, and that positive associations have been identified between patient satisfaction and patient compliance [8], patient preferences can be 
a

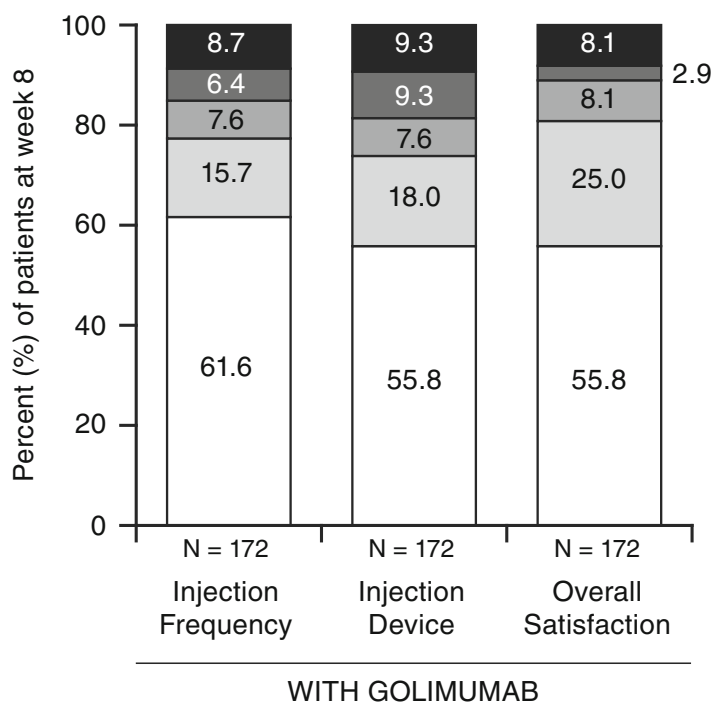

(PRIOR ANTI-TNF ADMINISTERED VIA SYRINGE)

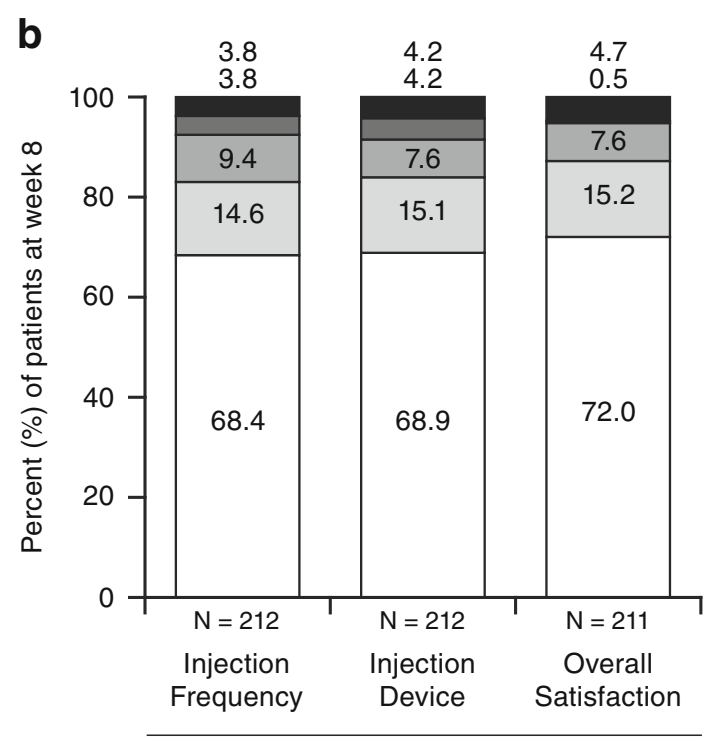

WITH GOLIMUMAB

(PRIOR ANTI-TNF ADMINISTERED VIA PEN)

Very satisfied

Somewhat satisfied nor dissatisfied

Somewhat dissatisfied

Very dissatisfied

Fig. 3 Patient satisfaction with golimumab injection at week 8 among patients whose prior anti-TNF agent (adalimumab/etanercept) was administered via a syringe versus $\mathbf{b}$ pen. $T N F$ Tumor necrosis factor

indicators of treatment success and should be considered during routine evaluations of treatment quality by physicians and population health decision makers.

To our knowledge, the golimumab GO-SAVE trial is the first to comparatively examine patient satisfaction among biologic products in the field of rheumatology. As part of the phase IIIb multicenter GO-SAVE trial of patients with RA with inadequate disease control despite treatment with etanercept or adalimumab, patient satisfaction with subcutaneous golimumab and its auto-injector was compared with prior biologic treatment using questionnaires developed by study investigators to understand the different injection experience attributes reported by patients when treated with golimumab versus adalimumab or etanercept, which are important considerations in a patient-focused approach in healthcare. In this trial, patients were asked explicitly about injection-related pain, discomfort, stinging, burning, and redness. This active surveillance was employed to ascertain patient experience in lieu of the passive collection of adverse event reports, which likely would have under-captured these symptoms.

Our results show that most patients with RA who transitioned to golimumab from adalimumab or etanercept were satisfied with their overall golimumab experience, including preference for golimumab and its auto-injector over their previous medication and injection device. Supportive data were derived from the smaller number of patients who received open-label subcutaneous golimumab through nearly 1 year of treatment, the majority of whom reported much less discomfort and redness, pain, stinging, and burning with the golimumab injection versus their previous anti-TNF medication injection, indicating a notably improved patient treatment experience. Analysis results also indicate that more patients prefer the golimumab injection device (auto-injector pen) when their previous anti-TNF medication was administered via a pen versus a syringe. These findings are consistent with the high acceptance observed in a multicenter study assessing a newly developed subcutaneous methotrexate autoinjector in patients with RA [11] as well as with study results that showed strong acceptance of adalimumab and etanercept autoinjection pens [12-14].

The data presented herein are limited by their derivation from a subset of patients with RA who were willing to transition from the subcutaneous anti-TNF formulations of adalimumab and/or etanercept to that of golimumab. Furthermore, we did not incorporate adverse event data, which could have served as an additional measure of patient pain/ discomfort, into these analyses. In addition, reimbursement was provided for golimumab but not for prior anti-TNF agents, and we neither evaluated nor controlled for the number of prior anti-TNF injections received before transition to golimumab. As noted previously, longer-term data are limited by the smaller number of patients retained at week 44 relative to baseline and week 8 . Additionally, the questionnaires were developed by study investigators and 
not developed based on satisfaction questionnaires employed by payers or hospitals to assess quality-based initiatives such as Consumer Assessment of Healthcare Providers and Systems (CAHPS). Finally, while the overall results suggest that patients preferred subcutaneous golimumab injections to adalimumab or etanercept, these results need to be interpreted with caution because of the open-label nature of the trial and potential bias toward the current treatment.

Nonetheless, given the focus of the Centers for Medicare $\&$ Medicaid Services on measurement of patient experience in specialty care, as recently outlined for oncologists [10], these data provide valuable information to rheumatologists to help ensure they are meeting the needs of their patients when considering which treatments will provide them with the best overall experience and, thus, optimal outcome.

In conclusion, most patients with $\mathrm{RA}$ receiving golimumab following inadequate response to adalimumab/ etanercept were satisfied with their overall golimumab experience, including its auto-injector, compared with their previous injection device.

Acknowledgements The authors thank Jennifer H. Lofland, PharmD, $\mathrm{MPH}, \mathrm{PhD}$, of Janssen Scientific Affairs, LLC for her work on earlier versions of this manuscript; Georgia Chao, MS, of Janssen Scientific Affairs, LLC for her statistical support; and Michelle L. Perate, MS, a medical writing consultant employed by Janssen for assistance with manuscript preparation and submission.

Author contributions All authors met the International Committee of Medical Journal Editors (ICMJE) criteria for authorship by (1) providing substantial intellectual contribution to the study's conception and design (RJD, LAE), data acquisition (RJD, LHB, JRC, LAE), or data analysis (KLT) and interpretation (RJD, LHB, JRC, LAE); drafting the article or revising it critically for important intellectual content (RJD, LHB, JRC, LAE, KLT); approving the final version to be published (RJD, LHB, JRC, LAE, KLT); and agreeing to be accountable for all aspects of the work in ensuring that questions related to the accuracy or integrity of any part of the work are appropriately investigated and resolved (RJD, LHB, JRC, LAE, KLT). The corresponding author is the overall guarantor of the work.

\section{Compliance with Ethical Standards}

The GO-SAVE trial (NCT01004432, EudraCT 2009-010582-23) was conducted according to the principles of the Declaration of Helsinki and International Committee on Harmonisation good clinical practices. The protocol was approved by each study site's governing ethical body, and patients provided written informed consent prior to the conduct of any study-related procedures.

Conflicts of interest Dr. Brent owns Johnson \& Johnson stock. Dr. Curtis has received funding for research, fees for consulting and/or honoraria from AbbVie, Amgen, BMS, CORRONA, Crescendo Bioscience, Janssen, Lilly, Myriad, Pfizer, Roche/Genentech, and UCB. Dr. Ellis is an employee of Janssen. Dr. Ellis owns stock in Johnson \& Johnson. Drs. Dehoratius and Tang were employed by Janssen at the time of manuscript preparation.
Open Access This article is distributed under the terms of the Creative Commons Attribution-NonCommercial 4.0 International License (http://creativecommons.org/licenses/by-nc/4.0/), which permits any noncommercial use, distribution, and reproduction in any medium, provided you give appropriate credit to the original author(s) and the source, provide a link to the Creative Commons license, and indicate if changes were made.

\section{References}

1. Crow R, Gage H, Hampson S, et al. The measurement of satisfaction with healthcare: implications for practice from a systematic review of the literature. Health Technol Assess. 2002;6:1-244.

2. Ingersoll KS, Cohen J. The impact of medication regimen factors on adherence to chronic treatment: a review of literature. J Behav Med. 2008;31:213-24.

3. Barton JL. Patient preferences and satisfaction in the treatment of rheumatoid arthritis with biologic therapy. Patient Prefer Adherence. 2009;3:335-44.

4. Poulos C, Hauber AB, González JM, et al. Patients' willingness to trade off between the duration and frequency of rheumatoid arthritis treatments. Arthritis Care Res (Hoboken). 2014;66:1008-15.

5. Bolge SC, Goren A, Tandon N. Reasons for discontinuation of subcutaneous biologic therapy in the treatment of rheumatoid arthritis: a patient perspective. Patient Prefer Adherence. 2015;9:121-31.

6. Huffstutter JE, Kafka S, Brent LH, et al. Clinical response to golimumab in rheumatoid arthritis patients who were receiving etanercept or adalimumab: results of a multicenter active treatment study. Curr Med Res Opin. 2017;33:657-66.

7. van Riel PL, van Gestel AM, Scott DL. EULAR handbook of clinical assessments in rheumatoid arthritis. Alphen Aan Den Rijn: Van Zuiden Communications, B.V; 2000. p. 1-54.

8. Farley H, Enguidanos ER, Coletti CM, et al. Patient satisfaction surveys and quality of care: an information paper. Ann Emerg Med. 2014;64:351-7.

9. Centers for Medicare \& Medicaid Services. Health insurance marketplace quality initiatives. http://www.cms.gov/Medicare/ Quality-Initiatives-Patient-Assessment-Instruments/QualityInitiatives GenInfo/Health-Insurance-Marketplace-Quality-Initiatives. html. Accessed 17 June 2015.

10. Centers for Medicare \& Medicaid Services. Oncology care model. http://innovation.cms.gov/initiatives/Oncology-Care/. Accessed 17 June 2015.

11. Hudry C, Lebrun A, Moura B, Zinovieva E, Backers O, HermanDemars H. Evaluation of usability and acceptance of a new autoinjector intended for methotrexate subcutaneous self-administration in the management of rheumatoid arthritis. Rheumatol Ther. 2017;4:183-94.

12. Borrás-Blasco J, Gracia-Pérez A, Casterá MD, Rosique-Robles JD, Abad J. Educational session as a tool to increase patient satisfaction of switching etanercept from the prefilled syringe to the autoinjection pen. Expert Opin Biol Ther. 2013;13:1103-8.

13. Borrás-Blasco J, Gracia-Pérez A, Rosique-Robles JD, Casterá MD, Abad FJ. Acceptability of switching adalimumab from a prefilled syringe to an autoinjection pen. Expert Opin Biol Ther. 2010;10:301-7.

14. Corominas H, Garcia-Diaz S, Sanchez-Eslava L, Fíguls R. Switching adalimumab from syringe to pen. Expected outcomes. Expert Opin Biol Ther. 2012;12:805-6. 